\section{TRANSFORMACIÓN DE MERCADOS MUNICIPALES DE MADRID. DE ESPACIO DE CONSUMO A ESPACIO DE ESPARCIMIENTO}

\author{
Luis Salinas Arreortúa ${ }^{1}$
}

\section{TRANSFORMATION OF MUNICIPAL MARKETS IN MADRID. FROM A CONSUMPTION ENVIRONMENT TO A LEISURE ENVIRONMENT}

\author{
Luis Salinas Arreortúa ${ }^{1}$
}

\section{Abstract}

Abstract: From a commercial perspective, gentrification -understood as a class and social restructuration process - can be observed as a procedure in which commercial activity is focused on high-income groups, the result being the substitution of this kind of activity in terms of the participation of traders and the supply of products - a situation that is clearly identified in municipal markets. In this way, there is a dissemination of a market-oriented "model" which, apart from meeting the food requirements of a given neighborhood, creates leisure areas. Such a transformation was preceded by a 
antecedida por la implantación de un discurso de deterioro del estado físico de las instalaciones así como del declive de la actividad comercial, como una justificación de una necesaria transformación del mercado. Se presentan dos casos de estudio. El primero es el mercado San Antón, como un espacio comercial que ha sido transformado y hoy en día es reconocido como un espacio que ofrece productos gourmet y servicio de gran restauración. El segundo caso es el mercado Los Mostenses, siendo un mercado municipal de abasto para consumo de barrio, donde se ha impuesto un discurso de deterioro y declive con el objetivo de transformarlo en un espacio de recreación.

\section{PALABRAS CLAVE: GENTRIFICACIÓN COMERCIAL; MERCADO MUNICIPAL; SAN ANTÓN; LOS MOSTENSES.}

Recibido: 24-04-2015

Aceptado: 18-01-2016

1 México. Maestro y Doctor en geografía por la Universidad Nacional Autónoma de México, con estudios en Relaciones Comerciales en el Instituto Politécnico Nacional. Investigador Asociado $C$ de tiempo completo en el Departamento de Geografía Social del Instituto de Geografía, Universidad Nacional Autónoma de México. Correo electrónico: luis_arreortua@hotmail.com. discourse that uses the deterioration of amenities and the decline of commercial activity as an excuse to transform the market. This paper offers two case studies. The San Anton market, which is an old commercial space that was transformed into a space focused on the provision of gourmet and restoration services. The second case refers to the Los Mostenses market, which through a discourse of deterioration and decline has been transformed into a leisure space.

\section{KEYWORDS: COMMERCIAL GENTRIFICATION; MUNICIPALIMARKET; SAN ANTON; LOS MOSTENSES.}

Received: 24-04-2015

Accepted: 18-01-2015

1 Mexico. Professor and PhD in Geography, National Autonomous University of Mexico with a specialization in Commercial Relations, National Polytechnic Institute. Research associate, Department of Social Geography at the Institute of Geography, National Autonomous University of Mexico. Email: luis_arreortua@hotmail.com 


\section{Introducción}

Una de las transformaciones urbanas que se aprecian en las últimas décadas son los procesos de sustitución de un sector de población por otro de mayores ingresos, reconfigurando el espacio urbano mediante inversión de capital y transformando las prácticas de consumo. Estas transformaciones se producen en un contexto de neoliberalismo urbano, en donde las ciudades generan las condiciones necesarias para la atracción de capital privado, en una constante competitividad urbana. Los análisis de este proceso de gentrificación, más allá de poder discutir críticamente las diferencias y similitudes que caracterizan a este proceso en diversas partes del mundo ${ }^{2}$, suelen prestar mayor atención a las transformaciones en espacios residenciales, asociándolas al origen del concepto, mientras que la gentrificación comercial ha sido poco trabajada ${ }^{3}$.

El proceso de gentrificación, entendido como una reestructuración de clase y de relaciones sociales en el espacio, no se limita únicamente al ámbito residencial. Relacionar gentrificación exclusivamente al ámbito residencial supone una concepción clásica del concepto ${ }^{4}$. Esto último deriva de la definición proporcionada por Glass en la década

2 Janoschka, Sequera y Salinas, 2014.

3 González y Waley, 2012, p. 5; Bridge y Dowling, 2001, p. 93; Romero y Lara, 2015, p. 191.

4 Salinas, 2013. de los sesenta del siglo XX: "Uno a uno, muchos de los barrios obreros de Londres han sido invadidos por las clases medias. Degradadas y modestas calles de antiguas caballerizas convertidas en viviendas y casas pequeñas - dos habitaciones arriba y dos abajo- fueron sustituidas, cuando sus contratos expiraron, por residencias elegantes y costosas. [...] Una vez que este proceso de 'gentrificación' comienza en un barrio, continúa rápidamente hasta que todos o la mayoría de los ocupantes de la clase trabajadora originales son desplazados, y se transforma el carácter social del barrio" ${ }^{\prime 5}$. Por esta definición, tradicionalmente los estudios sobre gentrificación se han centrado en transformaciones ligadas al uso residencial, no obstante, representa una visión que ha sido ampliada ${ }^{6}$. Actualmente, aceptando la premisa de Smith, la gentrificación forma parte de un proceso de urbanismo neolibe$\mathrm{ral}^{7}$. Por ello, considerando la gentrificación como la reconstrucción de clase en el espacio, se puede analizar no solo en el mercado de la vivienda, sino en distintos escenarios ${ }^{8}$, siendo uno de ellos la gentrificación comercial.

Por gentrificación comercial podríamos entender un proceso en el que la actividad comercial se transforma para satisfacer las necesidades de un sector de población de mayores ingresos,

\section{Glass, 2010, p. 7 (traducción del autor).}

6 González y Hodkinson, 2014, p. 94; Smith, 2012, p. 86.

7 Smith, 2001.

8 Janoschka, Sequera y Salinas, 2014. 
manifestándose desplazamiento de actividad comercial (comerciantes y productos). Hablamos de sustitución paulatina de comercios tradicionales por una mayor oferta de comercio orientado a las nuevas clases que llegan: yuppies, turistas y jóvenes "pioneros". En el comercio al por menor se puede presentar desde construcción de espacios de ocio y consumo -centros comerciales tipo malls-, hasta reestructuración de la actividad comercial en espacios que albergaban comercio tradicional, pudiendo traer como resultado, en mayor o menor medida, que los comerciantes sean sustituidos por personal contratado por empresas o comerciantes con mayor capacidad de inversión, que los productos ofrecidos sean diferentes -gourmet, delicatessen, marcas exclusivas, productos suntuarios- y por tanto los consumidores tienden a ser de mayor poder adquisitivo. Lo esencial en estas transformaciones es que la actividad comercial se establece entre y para un sector de población de mayores ingresos.

La gentrificación comercial ha tendido a ser analizada como a la gentrificación residencial, es decir, a partir del ciclo clásico de la desinversión-declive y la regeneración-reinversión ${ }^{10}$. Se puede presentar en escenarios que no necesariamente respondan a un deterioro, sino más bien se encuentra en un escenario potencial de mayor rentabilidad. Mediante cambios de uso de suelo y cambios en la oferta de

9 Romero y Lara, 2015.

10 González y Waley, 2012. productos y servicios destinados para un sector de población de mayores ingresos ${ }^{11}$. Y en tanto se teje esta relación entre la gentrificación residencial y comercial, suele atenderse la transformación comercial como parte de un proceso de gentrificación residencial, en términos de barrio. Importante es cuestionar si la gentrificación comercial es parte de un proceso más amplio de gentrificación residencial o es el inicio de un proceso de gentrificación, es decir, un catalizador de transformaciones más amplias. Esta situación se aborda como el contexto en el cual se están transformando los mercados de Madrid.

Las transformaciones en el ámbito comercial, en particular sobre la comercialización de productos básicos de primera necesidad, recae principalmente en las tiendas de autoservicio de grandes superficies (en adelante supermercados) y en los mercados minoristas municipales (o tradicionales), que son aquellos espacios comerciales en donde los vecinos de barrio acuden por productos de alimentos básicos, como son frutas, legumbres, carnes, pollos, pescados y mariscos, entre otros. Sin embargo, muchos de los estudios al respecto desarrollados desde la década de los noventa del siglo pasado, se han centrado en analizar la proliferación de los supermercados, mientras que los mercados municipales han sido poco atendidos,

11 Salinas, 2014. 
más aún, los trabajos versan sobre la caída de sus ventas por una fuerte competencia por parte de los supermercados ${ }^{12}$.

Más allá de los estudios sobre los impactos que ha generado la expansión de los supermercados en los mercados tradicionales, hay escasos trabajos que analicen la transformación de los mercados públicos desde la gentrificación comercial, tal como lo realizan González y Waley ${ }^{13}$ para el caso del mercado Kirkgate en Leeds, Inglaterra y Boldrini y Malizial4 ${ }^{14}$ para el caso de los mercados el Abasto y el Norte del Gran San Miguel de Tucumán, Argentina.

En España, con una fuerte tradición de mercados públicos, resulta sorprendente que hayan pocos trabajos sobre las transformaciones en los mercados, los cuales se han convertido en espacio turístico, de ocio y consumo elitista, fragmentando la territorialización de los mercados, es decir, borrando la concepción de mercado como abastecimiento de proximidad para residentes del barrio (por lo general, población de bajos ingresos) y como espacio de convivencia y reproducción social, tal como sucede con los mercados de San Miguel y San Antón en Madrid, La Ribera en Bilbao, Colón y Russafa

\footnotetext{
12 Casares y Rebollo, 1997; Medina y Álvarez, 2009; Schwentesius y Gómez, 2005 y 2006; Garza, 2009.

13 González y Waley, 2012.

14 Boldrini y Malizia, 2014.
}

en Valencia o Santa Caterina y La Boquería en Barcelona, por mencionar algunos.

Los mercados son espacios públicos donde se puede observar las relaciones sociales que en ellos se reproducen. Más allá de su principal objetivo implícito, compra y venta de productos de alimentación, se aprecian relaciones de proximidad, amistad y convivencia, lo que siempre ha resultado de importancia para el consumidor habitual, principalmente población adulta y adulta mayor. Sin embargo, en algunos mercados esas prácticas se están transformando, dirigiéndose hacia el visitante ocasional, turista y un sector de población de ingresos elevados, para satisfacer actividades de ocio y esparcimiento. Por otro lado, los mercados tradicionales, como espacios públicos, están en constante organización y refuncionalización, aunque habrá que diferenciar los procesos que modifican la dinámica e infraestructura interna del mercado debido a su evolución sociotemporal y aquellos que responden a un contexto de libre mercado, es decir, en la manera en que se insertan en las transformaciones regulatorias de la neoliberalización ${ }^{15}$ : apertura para la inversión privada, cambios en la ordenanza de los mercados, apertura a prácticas diversas e instalación de barras de degustación al interior de los mercados.

15 Brenner, Peck y Theodore, 2011. 
El objetivo de este trabajo es analizar las principales características que se manifiestan en las transformaciones de los mercados municipales de Madrid, en particular la imposición de un discurso de deterioro de las instalaciones físicas y, por tanto, el declive de la actividad comercial como una estrategia legitimadora para transformaciones en mercados municipales que presentan cualidades urbanas, dentro de las cuales destaca su localización en la ciudad. Esta transformación selectiva reproduce un modelo de mercado que, más allá de satisfacer necesidades de alimentación, se instaura como un espacio de ocio y esparcimiento.

Después de la introducción, el texto se divide en 4 partes. En la primera sección se exponen, de manera general, las transformaciones de los mercados municipales en España, evidenciando que en distintas ciudades se están transformando, convirtiéndose en espacios de recreación.

En la segunda sección se describen las características de los mercados municipales de Madrid y los programas públicos, en particular el Plan de Innovación y Transformación de los Mercados de Madrid 2003-2011, en el cual se puede apreciar las medidas que ha tomado el Ayuntamiento de Madrid respecto a los 47 mercados municipales.

En la tercera sección se analizan dos casos de estudio: el mercado San Antón y el mercado Los Mostenses. El mercado San Antón, como un espacio comercial que ha sido transformado y hoy en día es reconocido como un espacio que ofrece productos gourmet y servicio de gran restauración, en cuyo proceso de transformación hubo necesidad de reducir la cantidad de locales comerciales, de 56 a 26, produciendo desplazamiento de antiguos comerciantes. En el segundo caso, siendo un mercado municipal de abasto para consumo de barrio, se ha implantado un fuerte discurso de deterioro y declive, a pesar de que es un mercado cuya actividad comercial está en funcionamiento al 100\%. En dicho mercado hay un proyecto de transformación de capital privado financiado por el grupo inversor Triball, que pretende transformar el mercado en un espacio de esparcimiento, para que sea reconocido como un sitio turístico y emblemático de Madrid.

Por último, en las conclusiones se manifiesta que las transformaciones de los mercados tienen una estrecha relación con una escala espacial mayor, ya sea porque se localizan en barrios que están siendo transformados -gentrificados-, muestra de ello es el caso del mercado San Antón, o cuya localización hace susceptible la trasformación para la obtención de una mayor renta, como es el caso del mercado Los Mostenses. La transformación del mercado San Antón y el proyecto que pretende transformar el mercado de Los Mostenses tienen en común que los antecede la imposición de un discurso de deterioro, a partir del cual se busca la reproducción de un modelo de mercado, destinado, más que al consumo, al esparcimiento. 
Para la presente investigación se hizo una revisión bibliográfica de los trabajos realizados sobre los mercados tradicionales, destacando en particular aquellos que sustentaran el análisis bajo la gentrificación comercial. Para el caso de la situación de los mercados municipales en Madrid, se analizaron básicamente tres documentos: el Plan de Innovación y Transformación de los Mercados de Madrid (2003-2011), la Ordenanza de Mercados Municipales (2010) y el Plan de Fomento e Impulso del Pequeño Comercio y la Hostelería (2008-2011). Para obtener información sobre las transformaciones de los mercados casos de estudio se hizo un trabajo de campo, el cual incluyó 12 entrevistas a los gerentes de los mercados, a presidentes de la asociación de mercados y a comerciantes. La selección de estos dos casos de estudio obedece a que son mercados que están presentes en los debates públicos sobre la transformación de mercados en Madrid. El mercado San Antón, debido a que es uno de los mercados más recientes en cuanto a su transformación, el cual ha pasado de ser un mercado tradicional a un mercado gourmet. Mientras que el mercado de Los Mostenses es actualmente un mercado tradicional, con una dinámica comercial en total funcionamiento (es decir, no hay subutilización o declive de la actividad comercial), pero tiene en ciernes un proyecto de transformación hacia un mercado gourmet. De ambos mercados destaca su localización céntrica en Madrid, lo que nos lleva a plantear la gentrificación selectiva.

\section{Transformación de los mercados municipales en España}

Los mercados tradicionales de España se encuentran inmersos en un discurso predominante que expone una necesaria renovación y transformación para hacer frente a un proceso, aparentemente, de deterioro y declive frente al crecimiento de supermercados, a los cambios de hábitos de consumo y a un deterioro de las instalaciones derivado de la poca inversión pública y de la escasa participación de los locatarios ${ }^{16}$.

Esta concepción se intenta imponer en el imaginario colectivo, cuyo objetivo consiste en argumentar que los mercados tradicionales están pasando por un agudo deterioro y declive, razón por la cual se presenta como necesaria su rehabilitación y revitalización económica. O bien, que los mercados tradicionales se encuentran en una fase de cierta vulnerabilidad, es decir, al final de un ciclo evolutivo como formato comercial susceptible de

16 Juste, 1993; Puxeu, 1993; Siurana, 1993; Casares y Rebollo, 1997; Alonso, 2003 y 2008. 
replantearse su futuro más inmediato ${ }^{17}$. Si bien es cierto que son diversos los casos en que el mantenimiento de las instalaciones y la propia dinámica comercial no es la más conveniente para los locatarios como para los consumidores del barrio, se podrían discutir diversos elementos que mantienen ese escenario, como falta de inversión pública y contexto de crisis económica que ha afectado el poder adquisitivo de un sector de la población, repercutiendo en los propios ingresos de los locatarios que se ven indispuestos a invertir en los locales del mercado, entre otros factores. Sin embargo, las estrategias de transformación de los mercados parecieran ir más allá de afrontar dicha situación, y se pretende, en diversos casos, hacer de los mercados municipales un espacio gastronómico de ocio y turismo, tal como plantean políticos y funcionarios públicos, como el alcalde de Santander, Íñigo de la Serna, quien siguiendo el modelo de mercados como San Antón o San Miguel en Madrid, pretende hacer del mercado de Puertochico un espacio de ocio y turismo, conformándose como un "punto de atracción y dinamizador de los barrios"18.

Distintas son las estrategias que se han adoptado para realizar dicha transformación de los mercados: introducción de nueva actividad en grandes superficies como un supermercado (mercado El Olivar en Palma de Mallorca; mercado de Sants y mercado del Ninot en Barcelona, cuya reapertura se produjo en mayo de 2015; mercado San Antón y Alto de Extremadura en Madrid, entre otros), un gimnasio con piscina (mercado Chamberí en Madrid) o un centro de salud (mercado de San Fernando en Madrid), derribar total o parcialmente el mercado para volver a construir uno nuevo (San Antón y Torrijos en Madrid; Santa Caterina en Barcelona; Mercado Sur de Burgos, entre otros), hasta llevar el mercado tradicional a una nueva superficie comercial -centro comercial tipo mall- (Puerta Bonita en Madrid) o privatizar en su totalidad el mercado (San Miguel en Madrid; Mercado Sur en Burgos).

\section{Situación de los mercados tradicionales de Madrid}

Madrid cuenta con 47 mercados municipales, localizados en 17 distritos de los 21 en los que se divide Madrid, cuya propiedad corresponde al Ayuntamiento, quien celebra contratos de ocupación mediante concurso público para su concesión administrativa, es decir, hablamos de una gestión indirecta a través de una concesión administrativa

\footnotetext{
17 Juste, 1993, p. 11; Casares y Rebollo, 1997, p. 76; Alonso, 2003, p. 27.

18 Lemaur, 2013.
} 
municipal, la cual está regulada por la Ordenanza de Mercados Municipales (2010).

El discurso de deterioro, que suele estar presente en procesos de gentrificación residencial, se ha importado para implementarse como un discurso que justifica las transformaciones en los mercados ${ }^{19}$. Esto se observa en medios masivos de comunicación (radio y televisión), en medios impresos ${ }^{20}$ y en documentos oficiales de las autoridades locales. En el Plan de Innovación (2003-2011) se establece que hacia mediados de los años noventa del siglo $\mathrm{XX}$, "los mercados municipales comienzan a dar señales de declive y a mostrar claros signos de obsolescencia de sus estructuras en relación con las modernas tendencias de distribución comercial"21, razón por la que el Ayuntamiento de Madrid implementa el Plan de Innovación y Transformación de los Mercados de Madrid (2003-2011). En este plan se hace mención a una relación de trabajo con los comerciantes de los mercados a través de las asociaciones de comerciantes, que en muchos casos son los concesionarios de los mercados tradicionales, y con la Federación de Comercio Agrupado y Mercados de Madrid (COCAM), para conocer las necesidades y problemas de cada mercado y poner en marcha distintas propuestas para intervenir y transformar los distintos mercados.

19 González y Waley, 2012.

20 Pelayo, 2015.

21 Plan de Innovación (...), 2003.
Dicho plan contempló una inversión total de 142 millones de euros, de los cuales 51 millones han sido aportados por el Ayuntamiento de Madrid. La inversión se basó en tres líneas estratégicas de actuación: 1) el fomento de la renovación y modernización de las estructuras de los mercados, con una inversión de 114 millones de euros, de los cuales 48 millones los aportó el Ayuntamiento; 2) la mejora y promoción de una imagen en común, mercados de Madrid; y 3) la profesionalización de su gestión, en parte en cumplimiento con la ordenanza de mercados de 2010.

Además del Plan de Innovación, dependiente del Ayuntamiento de Madrid, la Comunidad de Madrid, mediante el Plan de Fomento e Impulso del Pequeño Comercio y la Hostelería (2008-2011) (Plan FICOH), de la Cámara de Comercio, ha invertido en diversos mercados tradicionales para su transformación.

Tanto el Ayuntamiento como la Comunidad de Madrid han apostado a la transformación de los mercados municipales, en la búsqueda de una salida de un aparente declive frente a los cambios en los hábitos de consumo y frente a las modernas tendencias de distribución comercial, lo que impone la idea del mercado tradicional como lo viejo, lo anticuado. Esta concepción del mercado tradicional se encuentra en la base del discurso legitimador de las transformaciones de los mercados tradicionales, para 
hacer, de algunos de ellos, espacios para el ocio y el turismo, destinados tanto para un sector de altos ingresos de Madrid como para turistas extranjeros.

Según el Plan de Innovación hay 20 mercados con una inversión mayor a un millón de euros, destacando el mercado de San Antón con 20,5 millones de euros (tabla 1), el cual contempló la demolición del antiguo mercado y la construcción de un espacio comercial con nuevas actividades: zona de degustación, un supermercado (Supercor), un restaurante con terraza y la incorporación de aparcamiento. Así también destaca la implantación de nueva actividad en 15 mercados tradicionales, en particular supermercados (Mercadona, Ahorramás, Hipercor, Lidl, Día, Eroski y Carrefour). Mientras que se han dejado 8 mercados sin intervención alguna (La Cebada, San Cristóbal, Vallehermoso, Valdezarza, Orcasur, Mediodía-Entrevías, Bami y Villaverde Alto).

De 2003 a 2011, periodo de mayor transformación de los mercados municipales de Madrid, hay elementos que caracterizan una situación particular de algunos mercados, los cuales dada su importancia se destacan a continuación: en el año 2008 el mercado Puerta Bonita fue trasladado al Centro Integrado de Vista Alegre únicamente dejando la planta baja para la oferta alimentaria tradicional, buscando como objetivo reunir actividades de ocio y consumo en un moderno espacio comercial, según declaraciones de la ex-presidenta regional Esperanza Aguirre y del ex-alcalde de Madrid Alberto Ruiz-Gallardón ${ }^{22}$. En 2009 fue privatizado el mercado San Miguel, el cual había sido remodelado en 1999 con recursos públicos. Mientras que el Nuevo Mercado de Torrijos, derrumbado en 2005, se reconstruyó en 2011 en un espacio en el cual se aloja, además del mercado, un centro comercial. En dicho proceso se transforma hacia una gestión privada (enajenando a la sociedad de comerciantes). En el año 2011 se había concluido la demolición del antiguo mercado de Barceló relocalizándolo en un mercado temporal, con una inversión de 23 millones de euros. Cabe preguntarse si al igual que el mercado San Miguel pudiera transformarse en un mercado privado.

En otro escenario se encuentran los mercados de San Fernando y La Cebada. El primero ha reabierto 20 locales comerciales con diversas actividades, coexistiendo la tradición y el alternativismo ${ }^{23}$ comercial (trueque, productos biológicos-orgánicos, comercio justo, etcétera). Por eso cabría preguntarnos si ilas alternativas de comercio son verdaderas alternativas a la reconversión comercial o soluciones de clase media? En el mercado de La Cebada, con el derribo del antiguo mercado modernista en 1958, los comerciantes se constituyeron como una sociedad cooperativa. En 2007 se convoca a un concurso público para la elaboración de un proyecto, el cual proponía

22 El Mercado de Puerta Bonita (...), 2008.

23 Fernández, 2012. 
la demolición del mercado y la construcción de uno nuevo abarcando el solar que ocupaba el polideportivo de la plaza ${ }^{24}$, todo ello con el fin de crear un centro comercial privado. Este proyecto generó mucha resistencia por parte de la sociedad cooperativa y además se planteaba su construcción en un contexto de crisis económica, factores que influyeron para que el proyecto no se haya realizado aún.

En esta coyuntura económica, en un contexto de neoliberalismo urbano, es importante plantearse: ¿cómo se concreta el proceso de transformación de los mercados tradicionales?, ¿la transformación de los mercados tradicionales es parte de un proceso barrial o se manifiesta como el desencadenante de las transformaciones en el barrio? y icuáles son las consecuencias en la reorganización socioespacial de los procesos de gentrificación comercial?

Una de las hipótesis que se manejan es que el regreso a la ciudad consolidada -tanto del capital como de la gente-, como parte de un proceso de reestructuración sociodemográfica y económica en las áreas centrales de las grandes ciudades, se ve acompañado de transformaciones urbanas en zonas selectivas, destinadas para un sector de la población de altos ingresos. Por ello se reestructuran espacios tanto residenciales como comerciales para satisfacer las necesidades de la población de altos ingresos. Tanto espacios comerciales como diversos mercados tradicionales, los cuales no se

24 Robles, 2009. limitaban solo a satisfacer necesidades de consumo sino que también fungían como espacios apropiados por población adulta y adulta mayor, se transforman en espacios de ocio y de esparcimiento dirigidos a un sector de población joven y de altos ingresos, en su mayoría turistas, cambiando la propia dinámica comercial de los mercados tradicionales, muchos de los cuales se localizan en los centros históricos de las grandes ciudades.

Diversas son las modificaciones que se realizan para la gestión de los mercados, pues en la Ordenanza de Mercados Municipales (2010) se permite la introducción de nuevas actividades y se conforma un Plan de Innovación, el cual contempla variadas intervenciones cuyo objetivo consiste no solo en hacer frente a condiciones de mantenimiento o salvaguarda de patrimonio arquitectónico (como en las intervenciones en las fachadas de los mercados), sino en una profunda transformación de su función, su sentido y su relación con la ciudad ${ }^{25}$.

Además, se establece que las transformaciones selectivas que acontecen en diversos mercados tradicionales son antecedidas por un discurso de deterioro-declive, y más que hacer frente a condiciones de poca inversión y mantenimiento de los mercados, las transformaciones comerciales giran en hacer de ellos un espacio de ocio y consumo elitista, tal como se verá para el caso del mercado Los Mostenses.

25 Bravo y Porretta, 2009, p. 3. 
TABLA 1. PRINCIPALES ACTUACIONES DEL PLAN DE INNOVACIÓN.

\begin{tabular}{|c|c|c|c|c|}
\hline Mercado & Inversión total* & $\begin{array}{l}\text { Inversión } \\
\text { subvencionable }(€)^{*}\end{array}$ & $\begin{array}{l}\text { Subvención } \\
\text { municipal }(€)^{*}\end{array}$ & Actuaciones derivadas del Plan de Innovación \\
\hline San Antón & 20,5 & $14,750,602$ & $4,425,181$ & $\begin{array}{l}\text { Demolición del antiguo mercado. Construcción del nuevo mercado. Reestructuración comer- } \\
\text { cial con implantación de nuevas actividades: zonas de degustación, supermercado Supercor y } \\
\text { restaurante con terraza. Incorporación de aparcamiento. }\end{array}$ \\
\hline \multirow[t]{2}{*}{ Ibiza } & \multirow[t]{2}{*}{9,1} & \multirow[t]{2}{*}{$4,105,925$} & \multirow[t]{2}{*}{$1,231,777$} & $\begin{array}{l}\text { Rehabilitación integral del edificio. Renovación de todas las instalaciones. Reubicación y } \\
\text { remodelación integral de locales. }\end{array}$ \\
\hline & & & & Reestructuración comercial con implantación de nueva actividad, supermercado Ahorramás. \\
\hline Puerta Bonita & 9,1 & $8,629,228$ & $2,588,768$ & $\begin{array}{l}\text { Construcción de un nuevo mercado integrado dentro del edificio del Centro Integrado de Vista } \\
\text { Alegre. Traslado al nuevo mercado de los comerciantes del antiguo mercado de Puerta Bonita. } \\
\text { Reestructuración comercial, con incorporación de nueva actividad, Supermercado Hipercor. }\end{array}$ \\
\hline \multirow{2}{*}{$\begin{array}{l}\text { Puente de } \\
\text { Vallecas }\end{array}$} & \multirow{2}{*}{8,4} & \multirow{2}{*}{$6,582,809$} & \multirow{2}{*}{$1,974,843$} & $\begin{array}{l}\text { Rehabilitación integral del edificio. Renovación de todas las instalaciones. Reubicación y } \\
\text { remodelación integral de locales. }\end{array}$ \\
\hline & & & & $\begin{array}{l}\text { Reestructuración comercial con implantación de nueva actividad, supermercado Mercadona. } \\
\text { Incorporación de aparcamiento. }\end{array}$ \\
\hline \multirow[t]{2}{*}{ Orcasitas } & \multirow[t]{2}{*}{7,8} & \multirow[t]{2}{*}{$6,035,003$} & \multirow[t]{2}{*}{$1,810,501$} & $\begin{array}{l}\text { Rehabilitación integral del edificio. Renovación de todas las instalaciones. Reubicación y } \\
\text { remodelación integral de locales. }\end{array}$ \\
\hline & & & & Reestructuración comercial con implantación de nueva actividad, supermercado Mercadona. \\
\hline \multirow{4}{*}{ Chamberrí } & \multirow{4}{*}{7,3} & \multirow{4}{*}{$3,838,739$} & \multirow{4}{*}{$1,149,308$} & $\begin{array}{l}\text { Rehabilitación y aislamiento de la cubierta. Refuerzo de las estructuras. Modernización de las } \\
\text { instalaciones generales. }\end{array}$ \\
\hline & & & & Restauración de acabados interiores y exteriores. \\
\hline & & & & Remodelación de diversos locales comerciales. \\
\hline & & & & Reestructuración e implantación de nueva actividad, gimnasio con piscina (Maná). \\
\hline \multirow{2}{*}{ Argüelles } & \multirow{2}{*}{6,9} & \multirow{2}{*}{$3,872,316$} & \multirow{2}{*}{$1,161,695$} & $\begin{array}{l}\text { Rehabilitación integral del edificio, con recuperación del estado original de la fachada. Renova- } \\
\text { ción de todas las instalaciones. }\end{array}$ \\
\hline & & & & $\begin{array}{l}\text { Reubicación y remodelación integral de locales. Reestructuración comercial con implantación } \\
\text { de nueva actividad, supermercado Ahorramás. }\end{array}$ \\
\hline \multirow{2}{*}{ San Enrique } & \multirow{2}{*}{6,1} & \multirow{2}{*}{$5,250,303$} & \multirow{2}{*}{$1,575,091$} & $\begin{array}{l}\text { Rehabilitación integral del mercado. Renovación de instalaciones. Reubicación y remodelación } \\
\text { integral de locales. }\end{array}$ \\
\hline & & & & $\begin{array}{l}\text { Reestructuración comercial con implantación de nueva actividad, supermercado Mercadona. } \\
\text { Incorporación de aparcamiento. }\end{array}$ \\
\hline \multirow{2}{*}{$\begin{array}{l}\text { Santa María De la } \\
\text { Cabeza }\end{array}$} & \multirow[t]{2}{*}{5,3} & \multirow[t]{2}{*}{$3,781,339$} & \multirow[t]{2}{*}{$1,134,402$} & $\begin{array}{l}\text { Rehabilitación integral de fachada y cubiertas. Restauración de acabados interiores. Renova- } \\
\text { ción de instalaciones generales, climatización y servicios. Remodelación de locales. }\end{array}$ \\
\hline & & & & Reestructuración comercial con implantación de nueva actividad, supermercado Mercadona. \\
\hline Guzmán el Bueno & 5,2 & $1,749,103$ & 524,731 & $\begin{array}{l}\text { Rehabilitación integral del edificio. Refuerzo de la estructura. } \\
\text { Renovación de todas las instalaciones. Reubicación y remodelación integral de locales. Reestructuración comer- } \\
\text { cial con implantación de nueva actividad, supermercado Ahorramás. }\end{array}$ \\
\hline
\end{tabular}

Nota: * millones de euros.

Fuente: elaboración propia con base en el Plan de innovación y transformación de los mercados de Madrid (2003). 


\section{Caso de estudio: San Antón y Los Mostenses}

\section{SAN ANTÓN, REPRODUCCIÓN DEL MODELO DE ESPARCIMIENTO}

El mercado de San Antón se localiza en el barrio de Chueca, en el centro histórico de Madrid (figura 1). Construido en 1945, se conformó como el espacio de abastecimiento de alimentación del barrio. En los ochenta se aprecian transformaciones económicas y sociales en el barrio, incluyendo aspectos socioculturales como la movida, que estimularon un entorno diverso cuya oferta de establecimientos comerciales - pubs o cafés de moda- se ampliaba a centros culturales, de ocio y entretenimiento ${ }^{26}$. Hacia la década de los 90, el barrio de Chueca se caracterizó por ser de esparcimiento y residencia de población gay, que junto a la oferta de establecimientos comerciales y de ocio revalorizaron el barrio. La transformación del barrio puede ser considerada como uno de los elementos que inciden en la decisión de transformar el mercado San Antón. En este caso, la transformación comercial del mercado responde a una escala mayor, a un proceso de gentrificación residencial en el barrio de Chueca.

En 1996 se entrega la concesión del mercado San Antón a la Asociación Profesional de Comerciantes

26

Imbert, 1986. del Mercado San Antón, integrada por los comerciantes del mercado. Esta asociación será la responsable de coordinar la transformación del mercado San Antón. Para la concesionaria, el mercado San Antón, localizado en pleno centro de Madrid y en un barrio que había presentado una creciente dinámica económica, no se correspondía con la dinámica del barrio, en particular con las preferencias de consumo de los residentes. Ahí es cuando se impone el discurso del deterioro del mercado: "Cuando empezamos con el proyecto había más locales, te puedo decir que entre el 2000 y el 2004 empezó a pegar [decaer] muchísimo el mercado, primero porque estaba muy sucio, estaba de verdad asqueroso; yo trabajaba en él y estaba asqueroso, y además porque muchos comerciantes ya no aspiraban a más" (entrevista con el gerente del mercado). La asociación de comerciantes decide concretar un proyecto para transformar el mercado, apoyados en el Plan de Innovación de los Mercados de Madrid, programa del Ayuntamiento de Madrid que apoyaba con hasta un 30\% de subvención para la inversión en los mercados municipales.

En el proceso de la creación del proyecto de transformación del mercado no todos los integrantes de la asociación de comerciantes estaban de acuerdo, pues la implementación del proyecto implicaba los compromisos derivados de la aceptación de un crédito otorgado por la empresa de distribución 


\section{FIGURA 1. LOCALIZACIÓN DE LOS MERCADOS MUNICIPALES DE MADRID.}

1 Alto Extremadura. 2 Antón Martín. 3 Argüelles. 4 Bami. 5 Barceló. 6 Chamartín. 7 Chamberí. 8 Diego de León. 9 Doña Carlota. 10 Embajadores. 11 Guillermo de Osma. 12 Guindalera. 13 Guzmán el Bueno. 14 Ibiza. 15 Jesús del Gran Poder. 16 La Cebada. 17 La Paz. 18 La Remonta. 19 Las Águilas. 20 Las Ventas. 21 Los Mostenses. 22 Maravillas. 23 Mediodía-Entrevías. 24 Moratalaz. 25 Numancia. 26 Orcasitas. 27 Orcasur. 28 Pacífico. 29 Prosperidad. 30 Puente de Vallecas. 31 Puerta Bonita. 32 San Antón. 33 San Cristóbal. 34 San Enrique. 35 San Isidro. 36 San Pascual. 37 Santa Eugenia. 38 Santa María de la Cabeza. 39 Tetuán. 40 Tirso de Molina. 41 Torrijos. 42 Usera. 43 Valdezarza. 44 Vallehermoso. 45 Vicálvaro. 46 Villa de Vallecas. 47 Villaverde Alto.

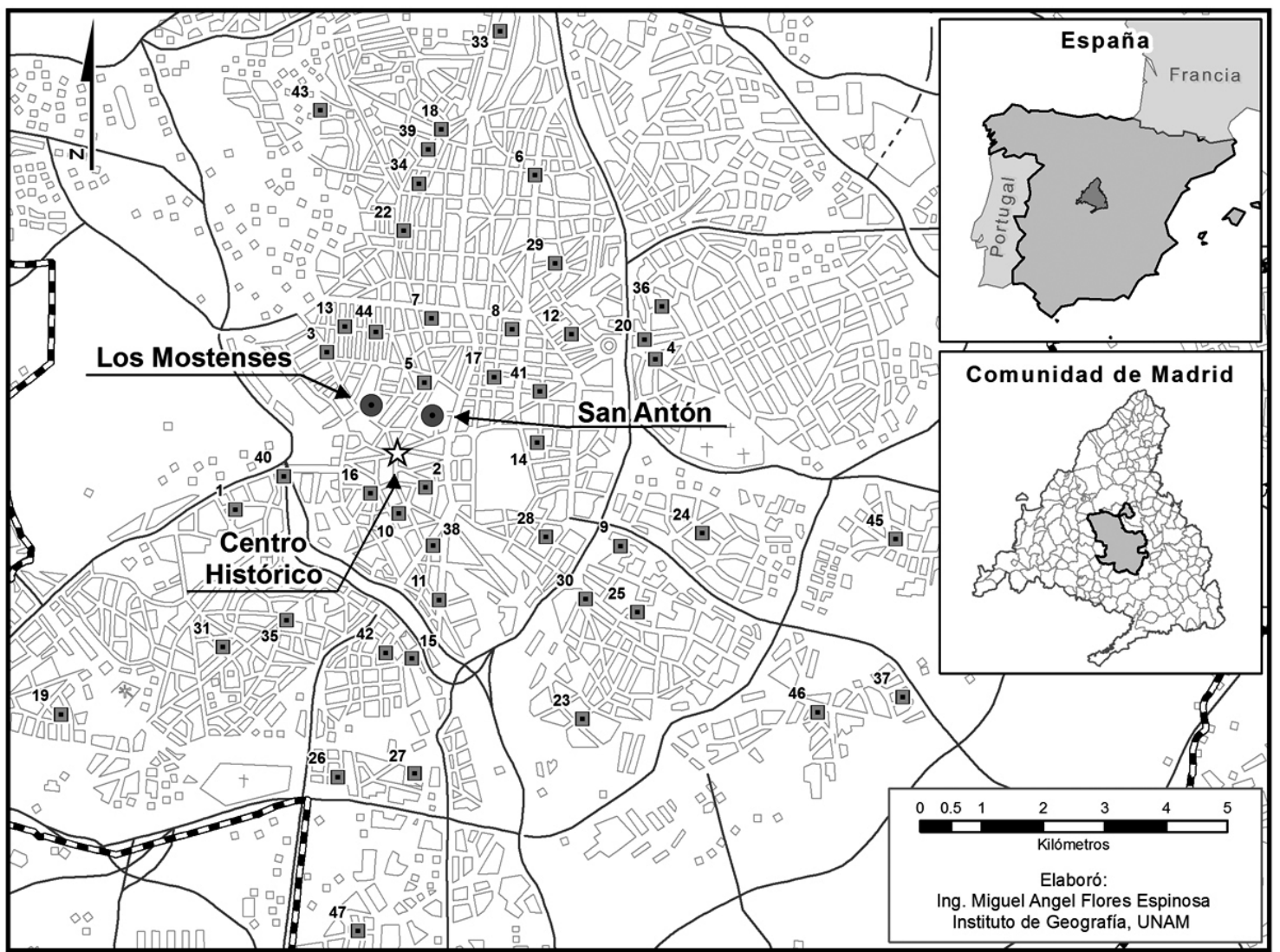

Fuente: Instituto Geográfico Nacional, Ministerio de Fomento e Instituto de Estadística, Comunidad de Madrid. 
Supercore del grupo El Corte Inglés, ya que el costo de la inversión ascendió a 20,5 millones de euros. Por esta razón, más de la mitad de los integrantes de la asociación decidieron no continuar, recibiendo una indemnización por su retiro de la asociación y los derechos que eso implicaba como comerciantes del mercado.

La transformación del mercado implicó que éste estuviera cerrado a partir del año 2007 hasta la inauguración del nuevo edificio el 17 de mayo de 2011 (figura 2). Desde ese año, la asociación está conformada por tan solo 6 integrantes, quienes alquilan a diversos comerciantes y empresas el espacio comercial del Mercado San Antón, conformado ahora con 26 locales comerciales ${ }^{27}$. La empresa que financió dicho proyecto hace uso de una tercera parte de la superficie comercial, estableciendo una tienda de autoservicio en la planta baja. En el primer piso, los locales ofrecen productos frescos, siendo una de sus características que cada local vende distintos productos (hay una charcutería, una pescadería, una frutería, una panadería, entre otros). El segundo piso se caracteriza por establecimientos de restauración (restaurant-bar con barras para degustación). Y el tercer piso alberga un restaurante-terraza.

El concepto del mercado San Antón implicó que tanto la ordenanza como distintas normas que regulaban las actividades de los mercados

\section{FIGURA 2. MERCADO SAN ANTÓN DESPUÉS DE SU TRANSFORMACIÓN.}

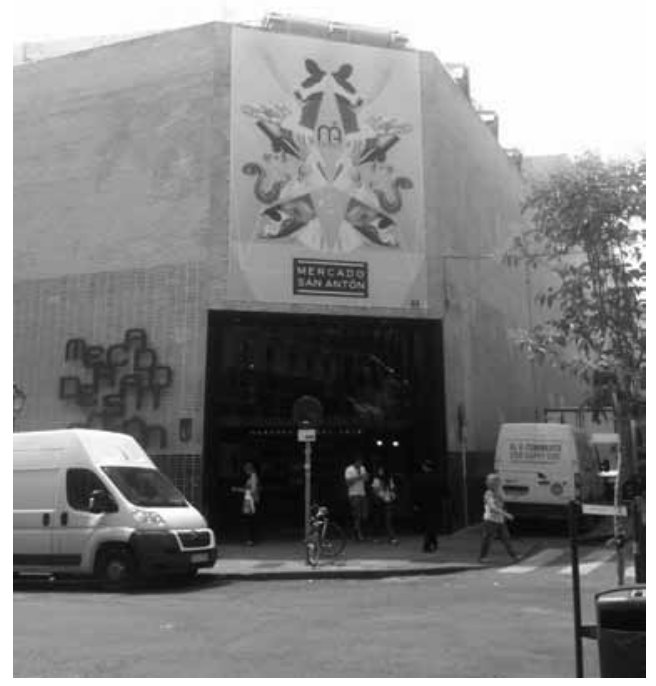

Fuente: autor, abril de 2014

municipales no contemplaran actividades que se presentaban en este mercado, como las zonas de degustación: "Nosotros hemos sido un proyecto innovador. Entonces, en las normativas, las ordenanzas, las leyes y en todo, no estaba contemplado un mercado de este tipo, un mercado municipal, entonces ha habido que saltarse..." (gerente del mercado San Antón). Lo que no quiere decir que las actividades se realicen de forma ilícita, pues la zona de degustación fue aprobada después de

27 El antiguo mercado municipal albergaba 56 locales comerciales. 
inaugurado el mercado. Esta situación muestra que las transformaciones comerciales responden a nuevas prácticas de consumo, ya que las zonas de degustación para turistas, las venta de productos gourmet y el bar-cafetería se instalan en espacios comerciales cuya actividad económica se transformó.

Los consumidores del mercado San Antón, además de provenir del barrio (gentrificado), se caracterizan por ser “...población profesional de poder adquisitivo medio alto que ha hecho de la distinción social de género su símbolo de identidad y éxito"28. Pero también atrae gente de otros barrios medios y altos de Madrid: "Viene más gente de afuera que del barrio, normalmente los fines de semana; y además el importe de las compras que hacen es bastante más elevado. Por ejemplo, gente que vive en Somosaguas, gente que vive en el centro de La Moraleja... cuando nos vamos al extrarradio se dejan compras de 200, de 300, de 400 y hasta de 800 euros" (gerente del mercado San Antón). Una de sus consecuencias en la reorganización socioespacial del barrio de Chueca ha sido la revalorización de la zona, en particular en los predios de uso residencial. Mientras que en los establecimientos comerciales aledaños (en particular bares y restaurantes tradicionales), el mercado representó una fuerte competencia, lo cual generó el cierre de unos y la (re)apertura de otros con ofertas de

28 Robles, 2013, p. 79. productos de diseñadores exclusivos (ropa y calzado) o productos selectos (vinos). Si la gentrificación residencial era una característica del barrio de Chueca, la transformación del mercado impactó en los establecimientos comerciales aledaños.

\section{Los Mostenses, imposición del discurso de deterioro}

El antiguo mercado Los Mostenses, erigido en 1875, formaba parte de los mercados modernistas de Madrid construidos a finales del siglo XIX y principios del XX, cuya edificación se caracterizaba por la utilización de hierro y cristal. Por distintas razones, algunos mercados modernistas fueron derribados, tales como el Mercado de Olavide o Chamberí, La Cebada y Los Mostenses (derribado en 1925 para la construcción de la Gran Vía). Actualmente se conserva el Mercado de la Paz y el Mercado de San Miguel${ }^{29}$.

En 1946 se construye por segunda ocasión el actual mercado (figura 3), localizado detrás de la Gran Vía y a un costado de la Plaza España, en el barrio centro de Madrid (figura 1). Este barrio ha pasado por cambios sociodemográficos relacionados en particular con un fuerte proceso de inmigración latinoamericana y asiática desde mediados de la década de los noventa del siglo XX. Este

29 Robles, 2009, p. 1. 


\section{FIGURA 3. ACTUAL MERCADO LOS MOSTENSES.}

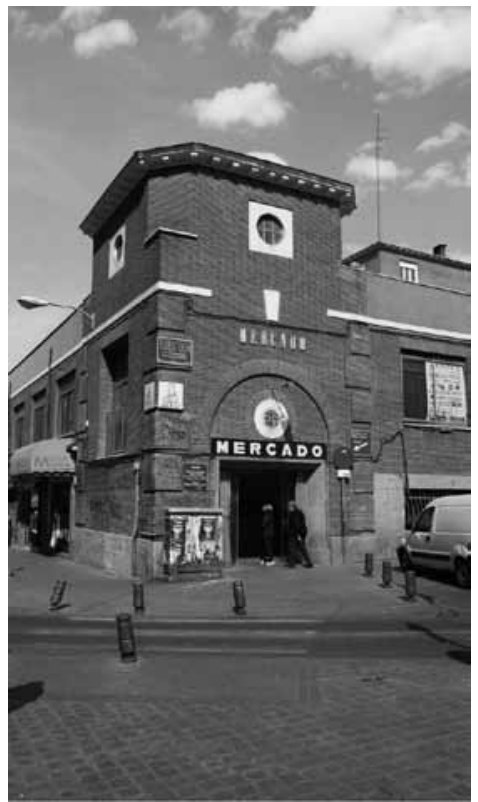

Fuente: autor, abril de 2014.

proceso social se expresa, entre otros aspectos, en la diversidad de la oferta de productos del mercado, la cual respondía básicamente a la población migrante. Hacia el año 2008, el 50\% de los adjudicatarios del mercado era de origen extranjero ${ }^{30}$.

Con la crisis de 2008 se aprecia una reducción de la población migrante, la que se ve en la necesidad

$30 \quad$ Robles, 2012. de buscar trabajo en otros países de Europa o de regresar a sus respectivos países. Según el Informe de la Población de Origen Extranjero Empadronada en la Comunidad de Madrid ${ }^{31}$, la tendencia de la población extranjera es a la baja; de 949.354 reportados como empadronados en la Comunidad de Madrid en enero de 2006, se llegó al máximo en enero de 2010 con 1.118.991 migrantes, a partir del cual se aprecia una disminución hasta llegar a 891.675 migrantes en junio de 2014.

A raíz de este proceso de movimiento de población, y en particular debido a la crisis económica, se comienza a imponer el discurso del deterioro del mercado, no solo en cuanto a su infraestructura sino en cuanto a la dinámica comercial del mercado. Es en este contexto en el cual se comienza a plantear la necesidad de implementar un proyecto para transformar el mercado.

A finales de 2010, la Oficina del Centro, dependiente de la Consejería de Urbanismo, encargó un proyecto a un despacho de arquitectura (Espejel y Fisac), el cual comprendía remodelar tanto el mercado como la plaza pública. Dicho proyecto consistiría en derribar el viejo mercado y levantar un edificio acristalado, de volúmenes apilados y 11 plantas de altura, con biblioteca, piscina, cine al aire libre, restaurante y un mirador, proyecto que costaría alrededor de 30 millones de euros ${ }^{32}$.

31 Información sobre evolución (...), 2014.

32 Sevillano, 2010. 
Dicho proyecto, además de que representaba una fuerte inversión, se enfrentó a una inconformidad por parte de los comerciantes, quienes solicitaban un mercado provisional en la Plaza España en vez de recibir indemnizaciones por el tiempo de duración de las obras, situación que el Ayuntamiento no aceptó $^{33}$.

Sin embargo, la importancia de la localización del mercado mantiene vivo el interés por su transformación. No obstante, se puede encontrar un fuerte contraste entre el discurso del deterioro con la dinámica comercial del mercado, pues hay una actividad comercial ocupada al 100\%, según datos proporcionados por el gerente del mercado y el presidente de la asociación de comerciantes, aunque se aprecia una falta de inversión, en particular para el mantenimiento de los locales e infraestructura del mercado, situación que no ha sido considerada por el Plan de Innovación y Transformación de los Mercados de Madrid (2003-2011). Lo anterior pareciera una estrategia de la administración pública de no apoyar a determinados mercados, que por su localización céntrica en la ciudad pudieran ser susceptibles de transformaciones comerciales. En esta situación se encuentran mercados como Los Mostenses, San Fernando y La Cebada.

El proyecto vigente para la transformación del mercado Los Mostenses es realizado por parte del grupo inversor Triball (Triángulo Ballesta), que se ha adherido a la asociación de comerciantes. El proyecto contempla la introducción de dos plantas destinadas a restaurantes con terrazas, además de remodelar los locales comerciales.

Según el gerente del mercado, quien ha sido el responsable de buscar la inversión del grupo Triball, el proyecto para transformar el mercado busca atraer población española de altos ingresos que gustan de ir a los cines, teatros y demás espectáculos musicales que se localizan en la Gran Vía: "Recuperar al cliente español es muy difícil porque se ha conseguido un nivel, dentro de la distribución de alimentación, muy alto en España, muy alto (...) El español está acostumbrado a entrar al centro comercial inmaculado, perfectamente colocado, sin olor, con una climatización espectacular (...) Recuperar al cliente español estando como está el mercado es imposible..." (entrevista al gerente del mercado).

Lo que se busca es transformar el mercado tradicional, y por tradicional se remiten a un mercado antiguo, como menciona el presidente de la asociación de comerciantes, quien quiere que el mercado de Los Mostenses se convierta en un mercado de siglo XXI: “...yo pienso que se puede abrir el abanico, aquí hay dos mercados que han empezado así como son San Miguel y otro en el barrio de Chueca

Villalba, 2010 y 2012. 
[se refiere a San Antón]. Aquí lo que queremos es a traer a gente de otro ámbito, por ejemplo, pues no es lo mismo la gente que compra aquí en el mercado porque su poder adquisitivo es menor, que luego empezar a atraer gente cuyo poder adquisitivo sea mayor, que al mismo tiempo va evolucionando, incluso los puestos (...). Abrir la puerta a una serie de establecimientos que antes no entraban, como puede ser la gran restauración..." (entrevista al presidente de la asociación del mercado).

Los representantes del mercado, integrantes de la junta directiva de la asociación y el gerente del mercado, buscan a toda costa la transformación del mercado lo cual implica, además de dirigir la actividad económica hacia población de mayores ingresos, la transformación de los productos, sin importar que una posible consecuencia sea la sustitución o el desplazamiento de comerciantes, por aquéllos que estén dispuestos a ofrecer productos diferentes, tal como ocurrió en la implementación del proyecto del mercado San Antón: "Van a tener que cambiar (los productos) obligatoriamente, además van a cambiar por el ritmo natural, nadie va a obligar a nadie..." (entrevista al gerente del mercado). Sin embargo, existe una importante diferencia entre los locatarios, quienes son integrantes de la asociación de comerciantes, pues consideran que lo que se busca es precisamente la transformación del mercado sin importar el reemplazo de los comerciantes actuales: "Lo que quieren es devorarnos; como no nos pueden echar porque no tienen derecho (...) lo que quieren es ahogarnos..." (entrevista a un locatario, integrante de la asociación del mercado).

El principal objetivo de la transformación del mercado de Los Mostenses es una reestructuración de clase dentro de un espacio comercial. Se busca crear espacios -restauración, bar, productos gourmet-dirigidos para población de altos ingresos, lo que implica pasar de un mercado tradicional a un mercado de siglo XXI, con o sin los comerciantes que integran actualmente la asociación de comerciantes del mercado Los Mostenses, tal como pasó con el mercado San Antón.

Esta transformación puede repercutir en la actividad comercial del barrio, pues actualmente hay todavía una marcada actividad dirigida para población migrante (productos alimenticios, restaurantes y bares, entre otros), que responde al consumo de la población que caracterizó el barrio. Empero, esta posible transformación junto al contexto de crisis económica podría repercutir en una sustitución paulatina de comercios tradicionales, por una mayor oferta de comercio orientado al consumo de nuevas clases medias. Con ello, esta posible transformación comercial puede constituirse como la punta de lanza de un proceso de gentrificación a mayor escala, incidiendo en el barrio centro de Madrid. 


\section{Conclusiones}

Es cierto que los mercados municipales pasan por situaciones difíciles, en parte debido a la crisis económica que ha afectado la capacidad de consumo, así como también por el crecimiento de las grandes superficies. Sin embargo, lo que se aprecia en este contexto es una transformación selectiva de mercados municipales hacia un modelo de mercado de siglo XXI, el cual se dirige a población de mayor poder adquisitivo.

Una reestructuración de clase y de relaciones sociales en espacios selectivos, en mercados municipales cuya localización los convierte en espacios potencialmente idóneos para consumo de población de altos ingresos y turistas. Esta situación ha sido considerada desde las políticas públicas, en particular con el Plan de Innovación, pues otorga un fuerte apoyo económico para la implementación de un proyecto, como sucedió en el mercado San Antón y anteriormente con el apoyo del Ayuntamiento de Madrid al mercado San Miguel, o bien, se niega el apoyo -Los Mostenses y La Cebadapara agudizar los problemas de mantenimiento y con ello se impone el discurso del deterioro para justificar la intervención privada.

Como se pudo observar para los casos de estudio tratados, las transformaciones a mayor escala han afectado las transformaciones de los mercados, muestra de ello es el caso del mercado San Antón.
Sin embargo, la coyuntura económica que afectó al barrio centro trajo como resultado la implementación de un discurso de deterioro, ya que el barrio se vio perjudicado por la crisis de 2008, razón por la cual se impone la necesidad de un proyecto que transforme el mercado Los Mostenses, y con ello pueda generarse una transformación a mayor escala.

Lo que se aprecia en ambos casos es la imposición de un discurso de deterioro. Para el caso del mercado San Antón, el mercado tal y como se encontraba no se correspondía con las prácticas del barrio, es decir, responde a un proceso mayor de gentrificación. Mientras que para Los Mostenses, las consecuencias de la crisis económica en el barrio da origen al discurso que establece la necesidad de transformar el mercado municipal, el cual se dirige hacia un consumo exclusivo, dirigido para un sector de altos ingresos. Con ello se esperaría una transformación a mayor escala. Esta reestructuración de clase y de relaciones sociales en mercados municipales de Madrid es característica del proceso de gentrificación comercial selectiva que está ocurriendo en determinados mercados de Madrid.

\section{Agradecimientos}

Este trabajo es realizado en una estancia de investigación posdoctoral realizada en la Universidad Autónoma de Madrid. Este texto está en el marco 
del proyecto de investigación "Contested_Cities - Contested Spatialities of Urban Neoliberalism: Dialogues between Emerging Spaces of Citizenship in Europe and Latin America". El autor agradece a Contested_Cities y a la Secretaría de Ciencia, Tecnología e Innovación del Distrito Federal, México, por el apoyo económico brindado para la realización de la estancia posdoctoral.

\section{Bibliografía}

ALONSO, Roberto. Modernización de los mercados municipales minoristas, servicio público y eficiencia empresarial. El papel de Mercasa. Distribución y consumo. 18(100): 168-174, 2008. ISSN 1132-0176.

ALONSO, Roberto. Los mercados municipales como producto establecimiento. Distribución y consumo. 13(69): 27-33, 2003. ISSN 1132-0176.

BOLDRINI, Paula y MALIZIA, Matilde. Procesos de gentrificación y contragentrificación. Los mercados de Abasto y del Norte en el Gran San Miguel de Tucumán (noroeste argentino). Revista INVI. 29(81): 157-191, 2014. ISSN 0718-8358. DOI $10.4067 / S 0718-83582014000200005$.

BRAVO, David y PORRETTA, Daniele. Elegía por los mercados de Barcelona. Masala Periòdic d’informació, denúncia i crítica social a Ciutat Vella. (49): 3, setembre 2009.

BRENNER, Neil; PECK, Jamie y THEODORE, Nik. ¿Y después de la neoliberalización? Estrategias metodológicas para la investigación de las transformaciones regulatorias contemporáneas. [En línea]. Urban. (1): 21-40, 2011. ISSN 2174-3657. Disponible en: http://polired.upm.es/index.php/ urban/article/view/409/1878.

BRIDGE, Gary y DOWLING, Robyn. Microgeographies of retailing and gentrification. Australian Geographer. 32(1): 93-107, 2001. ISSN 0004-9182. DOI $10.1080 / 00049180020036259$.

CASARES, Javier y REBOLLO, Alfonso. Mercados minoristas tradicionales. Situación actual y alternativas de actuación. Distribución y Consumo. 7(32): 75-114, 1997. ISSN 1132-0176.

EL MERCADO de Puerta Bonita formará parte del complejo comercial de Vista Alegre. [En línea]. Comunidad de Madrid. 2008. [Fecha de consulta: 10 de marzo de 2014]. Disponible en: http://www. madrid.org/cs/Satellite?c=CM_Actualidad_FA\&c $\mathrm{id}=1142496197086 \&$ language $=$ es $\&$ pagename $=C$ omunidadMadrid\%2FEstructura.

FERNÁNDEZ, Marta. Renovar un mercado de barrio, versión Lavapiés. [En línea]. El País. 2012. [Fecha de consulta: 10 marzo 2014]. Disponible en: http://ccaa.elpais.com/ccaa/2012/03/29/madrid/1333045590_721372.html.

GARZA, Laura. El mercado público ante el reto de la competitividad. [En línea]. CONCYTEG. 2009. [Fecha de consulta: 07 marzo 2014]. Disponible en: http://www.concyteg.gob.mx/formulario/MT/ MT2009/MT8/SESION3/MT83_LGARZA_137. pdf. 
GLASS, Ruth. London: aspects of change. En: LEES, Loretta, ed.; SLATER, Tom, ed. y WYLY, Elvin, ed. The gentrification reader. London, Routledge. 2010. ISBN 978-0-415-54839-7.

GONZÁLEZ, Sara y WALEY, Paul. Traditional Retail Markets: The New Gentrification Frontier? Antipode: a radical journal of geography. 45(4): 965-983, 2012. ISSN 1467-8330. DOI 10.1111/j.1467-8330.2012.01040.x.

GONZÁLEZ, Sara y HODKINSON, Stuart. Gentrificación como política pública en una ciudad provincial. El caso de la ciudad de Leeds en el Reino Unido. Revista Geografía Norte Grande. (58): 93-109, 2014. ISSN 0718-3402. DOI 10.4067/ S0718-34022014000200006.

IMBERT, Gerard. El Madrid de la "movida". [En línea]. El País. 25 de enero de 1986. [Fecha de consulta: 19 octubre 2014]. Disponible en: http://elpais.com/ diario/1986/01/25/opinion/506991609_850215. html.

INFORMACIÓN sobre evolución de la inmigración en Madrid. Comunidad de Madrid. 2014. [Fecha de consulta: 05 de noviembre de 2014]. Disponible en: http://www.madrid.org/cs/Satellite?pagenam $\mathrm{e}=$ PortalInmigrante/Page/INMI_pintarContenido Final\&cid=1142340982052.

JANOSCHKA, Michael; SEQUERA, Jorge y SALINAS, Luis. Gentrification in Spain and Latin America - a Critical Dialogue. International Journal of Urban and Regional Research. 38(4): 1234-1265, julio 2014. ISSN 1468- 2427. DOI 10.1111/1468-2427.12030.
JUSTE, Ángel. Mercados minoristas tradicionales. La remodelación necesaria. Distribución y Consumo. (11): 10-26, 1993. ISSN 1132-0176.

LEMAUR, V. El Mercado de Puertochico podría transformarse en un espacio de ocio y restauración. [En línea]. El Diario Montañés. 02 febrero 2013. [Fecha de consulta: 04 marzo 2014]. Disponible en: http://www.eldiariomontanes.es/20130202/ local/santander/mercado-puertochico-podriatransformarse-201302021307.html.

MEDINA, Xavier y ÁLVAREZ, Marcelo. El lugar por donde pasa la vida. Los mercados y las demandas urbanas contemporáneas: Barcelona y Buenos Aires. Estudios del hombre. (24): 183-201, 2009. ISSN 1405-1117.

PELAYO, Francisco. Una decena de mercados de barrio de Madrid agonizan por la crisis y la falta de reformas. [En línea]. 20 Minutos. 8 abril de 2015 [Fecha de consulta: 21 septiembre 2015]. Disponible en: http://www.20minutos.es/noticia/2408125/0/ botella/actuaciones-prometidas/ mercados-municipales/.

ROBLES, Juan. Mercado de San Antón. Madrid. Distribución y Consumo. (130): 77-82, 2013. ISSN 1132-0176

--- El mercado de los Mostenses (Madrid). Distribución y Consumo. (122): 110-114, 2012. ISSN 1132-0176.

--- Mercado de La Cebada (Madrid). Distribución y Consumo. (105): 141-146, 2009. ISSN 1132-0176.

ROMER0, Luis y LARA, Laura. De barrio-problema a barrio de moda: gentrificación comercial en 
Russa-fa, El "Soho" valenciano. Anales de Geografía de la Universidad Complutense. 35(1): 187-212, 2015. ISSN 0211-9803.

PLAN de innovación y transformación de los mercados de Madrid: 2003-2011 ocho años impulsando el comercio. [En línea]. Ayuntamiento de Madrid. 2003. [Fecha de consulta: 06 diciembre 2013]. Disponible en: http://www.madrid.es/UnidadesDescentralizadas/Consumo/ficheros/MercadosJunio2012.pdf.

PUXEU, Josep. Remodelación de mercados tradicionales. Modernización comercial y rehabilitación urbana. Distribución y consumo. (11): 32-35, 1993. ISSN 1132-0176.

SALINAS, Luis. Transformaciones urbanas en el contexto neoliberal. La colonia condesa en Ciudad de México: un proceso de gentrificación. [En línea]. Ci [ur] Cuadernos de Investigación Urbanística. (93), marzo 2014. ISSN 1886-6654. Disponible en: http://polired.upm.es/index.php/ciur/article/ view/2951.

SALINAS, Luis. Gentrificación en la ciudad latinoamericana. El caso de Buenos Aires y Ciudad de México. [En línea]. GeoGraphos. 4(44): 283- 307, febrero 2013. ISSN: 2173-1276. DOI 10.14198/ GEOGRA2013.4.44.

SCHWENTESIUS, Rita y GÓMEZ, Manuel. Supermercados y pequeños productores hortofrutícolas en México. Comercio Exterior. 56(3), 2006. ISSN 0185-0601.
--- Supermercados y su impacto sobre la comercialización de hortofrutícolas y pequeños productores en México. México, Centro de Investigaciones Económicas, Sociales y Tecnológicas de la Agroindustria y la Agricultura Mundial (CIESTAAM), Universidad Autónoma Chapingo. 2005. Serie Reportes de Investigación.

SEVILLANO, Elena. Los Mostenses tendrán que esperar. [En línea]. El País. 30 agosto 2010. [Fecha de consulta: 15 junio 2014]. Disponible en: http://elpais.com/diario/2010/08/30/madrid/1283167458_850215.html.

SIURANA, Antonio. Los mercados municipales ante el reto de la renovación. Distribución y Consumo. (11): 7, 1993. ISSN 1132-0176.

SMITH, Neil. La nueva frontera urbana. Ciudad revanchista y gentrificación. Madrid, Traficantes de sueños. 2012. p. 386.

--- Nuevo globalismo, nuevo urbanismo. Documents d'Anàlisi Geogràfica. (38): 15-32, 2001. ISSN 2014-4512.

VILLALBA, Enrique. Los comerciantes quieren unos Mostenses con terrazas. [En línea]. Madridiario. 21 mayo 2012. [Fecha de consulta: 15 junio 2014]. Disponible en: http://madridiario.es/ noticia/217311.

--- El nuevo mercado de los Mostenses, en suspenso. [En línea]. Madridiario. 20 agosto 2010. [Fecha de consulta: 15 junio 2014]. Disponible en: http:// madridiario.es/noticia/191019.

revista invi № 86 / May 2016 / Volume № 31: 179-201 201 
